\title{
In-channel integration of designable microoptical devices using flat scaffold-supported femtosecond-laser microfabrication for coupling-free optofluidic cell counting
}

\author{
Dong $\mathrm{Wu}^{1}$, Jian $\mathrm{Xu}^{1}, \mathrm{Li}-\mathrm{Gang} \mathrm{Niu}^{2}, \mathrm{Si}-\mathrm{Zhu} \mathrm{Wu}^{1,2}$, Katsumi Midorikawa ${ }^{1}$ and Koji Sugioka ${ }^{1}$
}

The high-precision integration of three-dimensional (3D) microoptical components into microfluidics in a customizable manner is crucial for optical sensing, fluorescence analysis, and cell detection in optofluidic applications; however, it remains challenging for current microfabrication technologies. This paper reports the in-channel integration of flexible two-dimensional (2D) and 3D polymer microoptical devices into glass microfluidics by developing a novel technique: flat scaffold-supported hybrid femtosecond laser microfabrication (FSS-HFLM). The scaffold with an optimal thickness of 1-5 $\mu \mathrm{m}$ is fabricated on the lower internal surface of a microfluidic channel to improve the integration of high-precision microoptical devices on the scaffold by eliminating any undulated internal channel surface caused by wet etching. As a proof of demonstration, two types of typical microoptical devices, namely, 2D Fresnel zone plates (FZPs) and 3D refractive microlens arrays (MLAs), are integrated. These devices exhibit multicolor focal spots, elongated (>three times) focal length and imaging of the characters 'RIKEN' in a liquid channel. The resulting optofluidic chips are further used for coupling-free white-light cell counting with a success rate as high as $93 \%$. An optofluidic system with two MLAs and a W-filter is also designed and fabricated for more advanced cell filtering/counting applications.

Light: Science \& Applications (2015) 4, e228; doi:10.1038/lsa.2015.1; published online 2 January 2015

Keywords: cell counting; 3D optofluidic chips; hybrid femtosecond laser microfabrication; microlens arrays; two-photon polymerization

\section{INTRODUCTION}

The integration of high-performance optical devices into microfluidics has recently attracted considerable attention due to unique optofluidic applications, ${ }^{1-3}$ such as microfocusing/imaging, optical trapping, fluid lasing and tunable optics, which are more challenging to achieve compared with the integration of non-photonic components into microfluidic systems due to requirements of high precision and surface quality. Certain conventional microfabrication technologies, such as soft ${ }^{4-6}$ or UV lithography, ${ }^{7}$ have been used to integrate two-dimensional (2D) microoptical devices, including optical waveguides, microring resonators, and distributed feedback dye lasers, with microfluidics. $^{2-8}$ These optofluidic chips afford a wide range of applications in light propagation, wavelength tuning and microfluidic lasing. The integration of three-dimensional (3D) optical micro/ nanostructures has further advanced the functionalization of optofluidic devices. For example, colloidal crystals with variable filtering effects integrated into a microfluidic chip by evaporation-induced self-assembly has resulted in space-selective transmission and blocking of particular wavelengths. ${ }^{9}$ Periodic photonic units with a bandgap have been incorporated in microfluidic channels by laser interference lithography to perform refractive sensing. ${ }^{10}$ However, these methods are only suitable for the preparation of specific periodic $3 \mathrm{D}$ microstructures due to inherent fabrication limitations and thus suffer from low flexibility and weak designability.

Two-photon polymerization (TPP) ${ }^{11-15}$ based on laser direct writing is a powerful technology for the fabrication of flexible $3 \mathrm{D}$ structures in a controllable manner with high precision $(<100 \mathrm{~nm})$ by point-to-point scanning. This method has been used to integrate various 3D functional microdevices, ${ }^{16-20}$ such as a microfilters, ${ }^{16}$ mixers ${ }^{18}$ or overpasses, ${ }^{19}$ into a $2 \mathrm{D}$ microchannel for controllable filtering of particles, high-efficiency mixing of different solvents, and guiding of different fluids and cell migration, respectively. However, all these works concern the integration of mechanical devices or 3D models in microchannels. To date, there have been no reports on the TPP integration of high-precision 2D-3D designable microoptical devices. Our group has reported a series of studies on the fabrication of $3 \mathrm{D}$ embedded glass microchannels using femtosecond laser-assisted etching (FLAE). ${ }^{21-23}$ The surface smoothness of the microchannels was

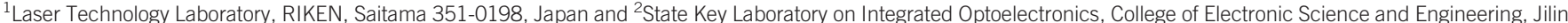
University, Changchun 130012, China

Correspondence: K Sugioka, Laser Technology Laboratory, RIKEN, 2-1 Hirosawa, Wako, Saitama 351-0198, Japan

E-mail: ksugioka@riken.jp

Received 9 June 2014; revised 23 September 2014; accepted 8 October 2014; accepted article preview online 7 november 2014 
improved by smaller-pitch line-to-line scanning and optimized postthermal annealing. To enhance their functionalities, some $1 \mathrm{D}$ to $3 \mathrm{D}$ glass microcomponents, including waveguides, ${ }^{24-26}$ microrotators, ${ }^{27}$ micromirrors, ${ }^{23}$ and microlenses, ${ }^{28}$ have been integrated into the channels. However, the FLAE method suffers from low fabrication precision, on the order of $10 \mu \mathrm{m}$, determined by the wet etching process. ${ }^{29}$ To compensate for this weakness and achieve more functional microchips, we have recently demonstrated TPP integration of $3 \mathrm{D}$ polymer devices into $3 \mathrm{D}$ glass microchannels prepared by FLAE using hybrid femtosecond laser microfabrication. ${ }^{30}$ The fabricated microchips are called ship-in-a-bottle biochips and have been used for successive filtering, mixing and synthesizing. A number of groups have also demonstrated the integration of functional polymer microcomponents, such as $3 \mathrm{D}$ microporous constructs ${ }^{18}$ and microfilters, ${ }^{20}$ into the closed glass microfluidic channels, which are prepared by non-laser technology. Our FLAE provides the advantage over these works that an identical femtosecond laser 3D micromachining system can successively implement both FLAE (subtractive manufacturing) and TPP (additive manufacturing), leading to more cost-effective and flexible fabrication of functional biochips. Specifically, FLAE can create 3D microfluidic structures with an almost arbitrary geometry (even multilayered structures) and cross-sectional shapes, and then, TPP can flexibly integrate various 3D functional microcomponents at any positions, ${ }^{30}$ which provides more $3 \mathrm{D}$ biomimetic environments for cell culture and other biological applications. However, due to the complex environment in the microchannel and high demand of high optical quality, TPP integration of 3D microoptical components into microfluidics, especially into 3D embedded microchannels, has never been demonstrated and remains a great challenge. Microoptical components require high surface smoothness to guarantee their optical performance compared with other microcomponents. ${ }^{16-20,30,31}$ Moreover, the flatness of the internal surfaces of the microchannels on which the components are created significantly affects their quality. Over areas measuring a few dozen microns on a side, the surfaces fabricated by FLAE have adequate optical quality. In contrast, the undulations over longer length scales need to be reduced.

In this paper, we report in-channel integration of designable highprecision microoptical devices for the first time, including 2D Fresnel zone plates (FZPs) and 3D refractive microlens arrays (MLAs) in 3D microchannels embedded in a glass substrate. To ensure the creation of high-performance microoptical devices, the lower internal surface of a microfluidic channel must be reconstructed to be highly flat over a long range. To accomplish this task, flat scaffold-supported hybrid femtosecond laser microfabrication (FSS-HFLM) is proposed. This process consists of the construction of a flat polymer scaffold within the glass microfluidic channel on the lower internal surface of a microfluidic channel, followed by the creation of microoptical devices on the scaffold, both accomplished by TPP. High-quality integration is achieved by the optimal design of a layer thickness of the scaffold (with 1-5 $\mu \mathrm{m}$ thickness) to eliminate surface undulations on the glass microchannel. The focusing and imaging of a $2 \mathrm{D}$ FZP and a $3 \mathrm{D}$ MLA in the channel in a liquid environment are investigated and compared with those on a flat surface in air. By this technique, 3D optofluidic devices based on a new concept were fabricated, which enable us to perform parallel cell detection and counting in a relatively wide channel using coupling-free white light. Thus, this paper presents two crucial novelties from the point of view of science and applications of lights, one is development of FSS-HFLM to integrate high performance polymer microoptics in channels and the other is demonstration of optical sensing of biosamples by using a new type of optofluidic devices (some other progresses as compared with previous works are summarized in Supplementary Table S1).

\section{MATERIALS AND METHODS}

\section{FSS-HFLM integration of high-precision 2D-3D microoptical} devices into $3 \mathrm{D}$ embedded glass microchannels

A 522-nm femtosecond laser beam was obtained by second-harmonic generation from an amplified femtosecond Er-fiber laser (FCPA $\mu$ Jewel D-400 (from IMRA America, Inc. Headquarters and Manufacturing, 1044 Woodridge Ave, USA.) with a wavelength of $1045 \mathrm{~nm}$, a pulse width of $360 \mathrm{fs}$, and a repetition rate of $200 \mathrm{kHz}$ ). A 3D embedded glass microchannel with high surface smoothness was prepared in a commercially available photosensitive Foturan glass (from Mikroglas, Langen (Hessen), Germany) by FLAE. ${ }^{21-23}$ The glass microchannel was filled with the epoxy-based negative-type resin SU8 (2075; MicroChem, Newton, America), which is widely used for FSS-HFLM fabrication (Supplementary Fig. S1). A layer of scaffold with an optimal thickness $(1-5 \mu \mathrm{m})$ was formed by femtosecond laser writing to eliminate the unflattened internal glass surface of the microfluidic channel in a long range caused by wet etching (Supplementary Figs. S2 and S3). The scanning was performed from the inner part of the glass below the bottom surface of the channel to the inner part of the channel. In general, the lateral size and pitch of laser scanning for constructing the flat scaffold were the same as for the optical devices. For fabrication of the flat scaffold as well as microoptical devices, a smaller scanning pitch $(200 \mathrm{~nm})$ was adopted to ensure a high surface smoothness (average roughness $\sim 2.5 \mathrm{~nm}$ ) compared with $500 \mathrm{~nm}$ for the filter/mixer fabrication used in our previous work. This alteration enables high-quality subsequent integration of microoptical devices onto the scaffold. In the laser-writing step, the data program for creating the scaffold was integrated with that for creating the microoptical device. Therefore, both the scaffold and the microoptical device could be fabricated by one-step laser scanning. The scaffold fabrication does not increase the complexity of the entire process. The total thickness of the scaffold and the $175-\mu \mathrm{m}-$ diameter amplitude-type FZP was $3 \mu \mathrm{m}$, which is much larger than that of a common flat-surface phase-type FZP, which has a thickness of less than $0.5 \mu \mathrm{m} .{ }^{31}$ For a single microlens $(40-\mu \mathrm{m}$ diameter) and seven microlens (280- $\mu \mathrm{m}$ diameter) array integration, $1-\mu \mathrm{m}-$ and 5 $\mu \mathrm{m}$-thick scaffolds were prepared, respectively. The 2D FZP and 3D MLA were integrated into the 3D glass microchannel using point-topoint laser scanning on the scaffolds. The FSS-HFLM did not obviously increase the experimental complexity while solving the problem of the undulated channel surface for the fabrication of high-quality microoptics.

\section{Optical characterization and biological application of 3D integrated optofluidic microchips}

A homemade optical characterization system (Supplementary Fig. S4) was developed to characterize the optical properties of the 3D integrated optofluidic chips. The system consisted of a halogen lamp, a CCD, a $50 \times$ objective lens, a pump and several plastic tubes. Under illumination from a halogen lamp, the focal spots and imaging are magnified by the objective lens and collected by a CCD. The pump is used to produce a negative pressure in the $3 \mathrm{D}$ biochip to suck the solvent from the beaker. Solvents containing cells can then be introduced into the biochip. The flow speed is adjustable from 0 to $5 \mathrm{~mm} \mathrm{~s}^{-1}$ by regulating the negative pressure. The halogen lamp, CCD, and objective lens can perform in situ monitoring of the cells for detection and counting. 
Fabrication and characterization of 3D optofluidic microsystems with two MLAs and a W-filter

Three polymer devices consisting of two MLAs and a W-filter were integrated into a 3D embedded glass microchannel one by one in a single laser direct-writing procedure. After development and drying, the $3 \mathrm{D}$ optofluidic system was characterized. Solvents containing cells of different sizes $(6-8 \mu \mathrm{m})$ were introduced into the optofluidic chip. Small cells with $50-\mu \mathrm{m}$ lengths and $6-\mu \mathrm{m}$ widths passed through the filter, whereas bigger cells with $72-\mu \mathrm{m}$ lengths and 8 - $\mu \mathrm{m}$ widths were hindered by the $\mathrm{W}$-filter, as observed in Supplementary Movie 2.

\section{RESULTS AND DISCUSSION}

\section{FSS-HFLM integration of 2D diffractive FZPs into 3D} glass microchips

Figure 1a presents a schematic illustration of a designed binary diffractive FZP that consists of six pairs of concentric odd and even zones. The outer radius of the $n$th zone from the center $\left(R_{2 n-1}\right.$ for an odd zone and $R_{2 n}$ for an even one) is determined by the formula

$$
R_{n}{ }^{2}+f^{2}=(f+n \lambda / 2)^{2}
$$

such that

$$
R_{n}=\sqrt{n \lambda f+(n \lambda / 2)^{2}}
$$

where $\lambda$ is the vacuum wavelength of light and $f=R_{1}^{2} / \lambda$ is the primary focal length. The outer radius of the first odd zone $R_{1}$ and of the sixth even zone $R_{12}$ are designed as $25.3 \mu \mathrm{m}$ and $87.6 \mu \mathrm{m}$, respectively, based on the channel width of approximately $280 \mu \mathrm{m}$. It is well known that the binary diffractive optics exhibit many advantages, such as low weight, small volume and easy fabrication by standard photolithography due to their $2 \mathrm{D}$ geometry. Two-photon polymerization has realized various amplitude- and phase-type FZPs on flat glass substrates ${ }^{32}$ due to its high flexibility (Supplementary Fig. S1b). However, in 3Dembedded microchannels, an undulated bottom surface caused by wet etching limits the availability of FZP integration. Even an irregularity as small as $1 \mu \mathrm{m}$ makes complete fabrication of an FZP impossible (Supplementary Fig. S5) because the usual thickness of an FZP is $0.5-1 \mu \mathrm{m} .{ }^{30}$ To eliminate this irregularity, a flat polymer scaffold with an optimal thickness (cf. Supplementary Fig. S2) is designed and constructed in the glass microchannel by TPP. According to SEM measurements, the channel irregularity is approximately $2 \mu \mathrm{m}$, such that the total thickness of the FZP and scaffold must be $3 \mu \mathrm{m}$, as demonstrated in Figure 1b. A thicker FZP/scaffold ( $5 \mu \mathrm{m}$ or larger) results in the collapse or floating of some outer zones during the development because their widths are much smaller than the thickness. Using this FSS-HFLM technique, high-quality polymer amplitude-type FZPs can be created on the scaffold inside the 3D glass microchannels (Figure 1c and Supplementary Fig. S1a). Under light illumination, the FZP should produce a round focal spot by optical diffraction, as illustrated in Figure $1 \mathrm{~d} .{ }^{33}$ Figure $1 \mathrm{e}$ and $1 \mathrm{f}$ present an SEM image of the polymer FZP on a flat surface and an optical microscopy image of two polymer FZPs in a $3 \mathrm{D}$ embedded glass channel, respectively. There are no missing or deformed parts for either FZP, and the FZPs conform to the design model even inside the microchannel.

Following FSS-HFLM integration of an FZP into a glass microchannel, the optical properties were systematically examined using a homemade characterization system (Figure 2f). When a 632-nmwavelength laser illuminated the FZP in an ethanol environment, a bright focal spot was observed, as shown in the inset of Figure 1g. The point spread functions were measured by extracting the grayscale
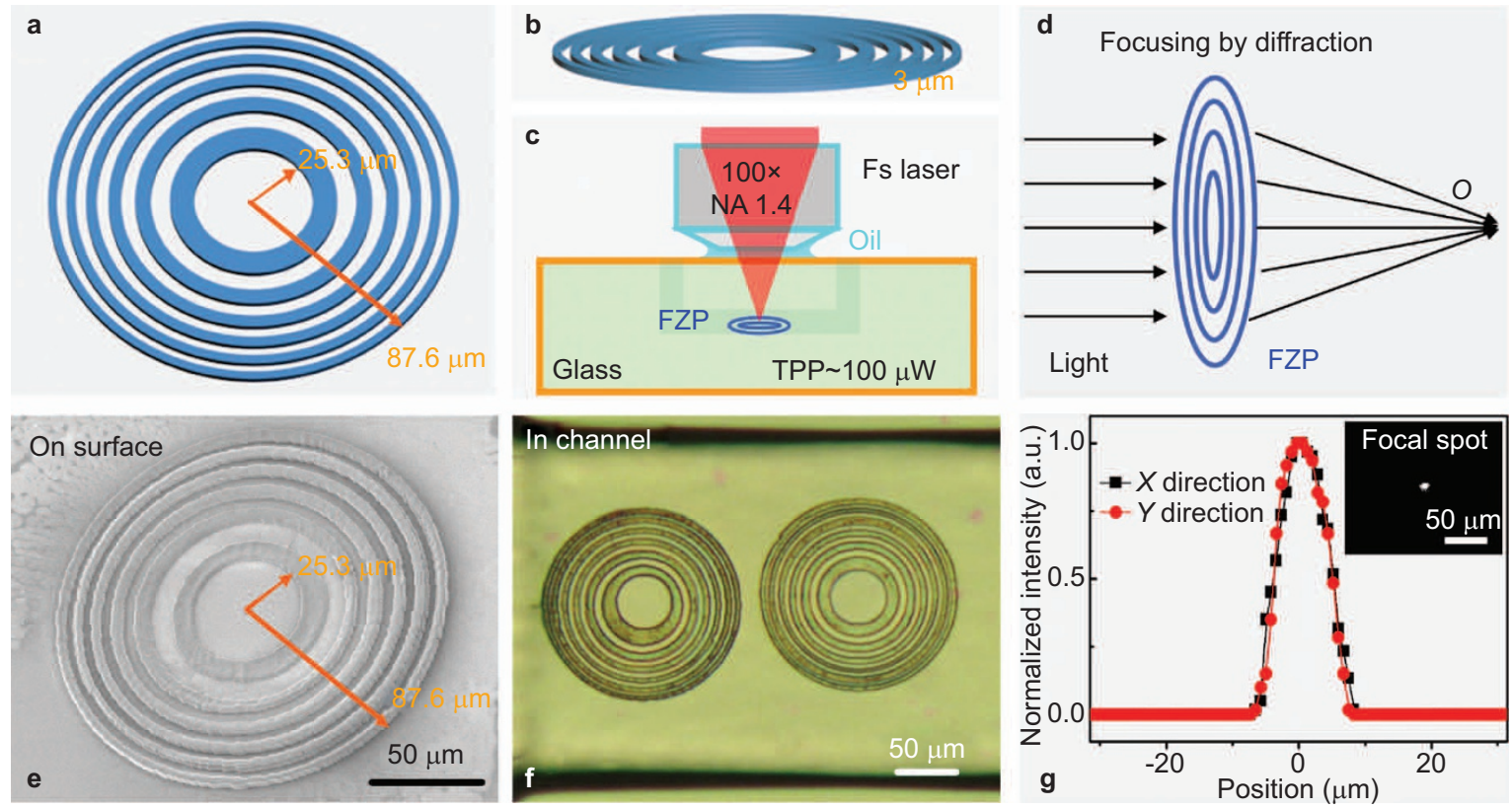

Figure 1 High-quality integration of a 2D diffractive FZP into a 3D embedded glass microchannel by FSS-HFLM. (a) $15^{\circ}$ and (b) $80^{\circ}$ tilted schematics of the designed FZP consisting of six pairs of odd and even zones. The outer zone radii of the first even zone and of the sixth even zone are $25.3 \mu \mathrm{m}$ and $87.6 \mu \mathrm{m}$, respectively. The 3 $\mu \mathrm{m}$-thick flat scaffold eliminates the slightly undulated channel caused by wet etching. (c) Illustration of the FSS-HFLM integration of a polymer FZP into a 3D glass microchannel. (d) The focusing of incident light by an FZP. (e) $15^{\circ}$ tilted SEM and (f) Optical microscopy images of a 175- $\mu \mathrm{m}$ FZP fabricated by FSS-HFLM on the surface and in the channel, respectively. (g) The focal spot and its grayscale intensity. The curves are extracted from the focal spot (shown in the inset) measured under 632-nm laser illumination, exhibiting nearly the same FWHM $(8.9 \pm 0.3 \mu \mathrm{m})$ along the $X$ and $Y$ directions. 2D, two-dimensional; 3D, three-dimensional; FSS-HFLM, flat scaffold-supported hybrid femtosecond laser microfabrication; FZP, Fresnel zone plate; NA, Numerical aperture; TPP, Two-photon polymerization. 

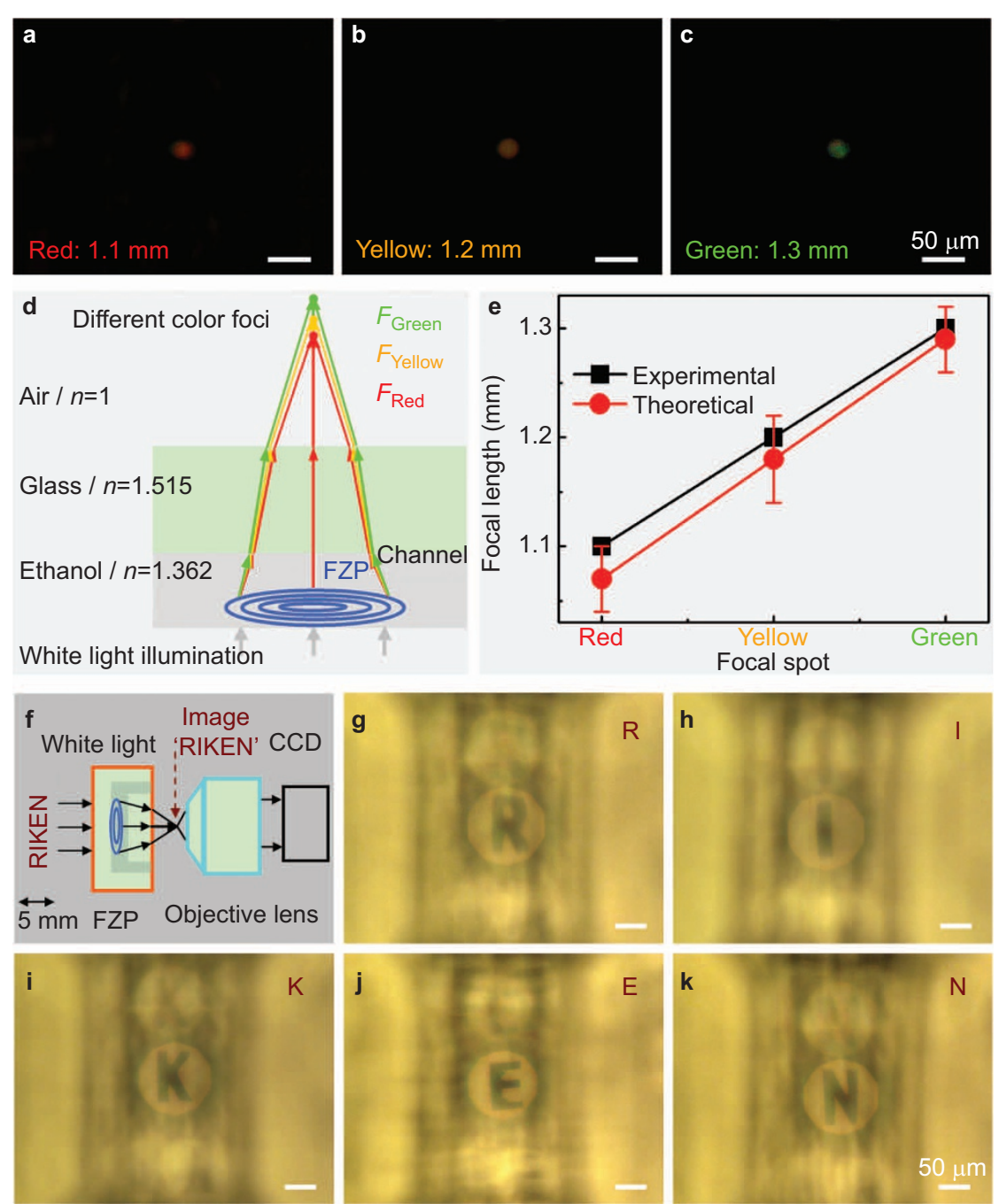

Figure 2 Optical properties of an FZP integrated in a microchannel in a liquid environment. (a-c) Optical microscopy images of focal spots for different colors, namely red, yellow and green. (d) The schematic image of different focal lengths $\left(F_{\text {red }}, F_{\text {yellow }}\right.$ and $\left.F_{\text {green }}\right)$ in ethanol under white light illumination. (e) Experimental and theoretical focal lengths for different colors in ethanol. (f) The homemade optical characterization system. The five letters 'RIKEN' of 5-mm size are placed in front of the optofluidic chip with an integrated FZP. The image is magnified by an objective lens and collected by the CCD. (g-k) Resulting images of the letters 'RIKEN'. FZP, Fresnel zone plate; CCD, Charge coupled device.

intensity of the focal spot, as plotted in the main graph in Figure 1g. The full-width at half-maximum (FWHM) of the focal spot sizes along the $X$ and $Y$ axes are nearly the same $(8.9 \pm 0.3 \mu \mathrm{m})$. Another optical feature of FZP is that it can produce focal spots with different colors under white light illumination (Figure 2d). Longer wavelengths result in shorter focal lengths. Therefore, the focal spots for different colors are observed at different positions (Figure $2 \mathrm{a}-2 \mathrm{c}$ ), as expected. The measured focal lengths were $1.1 \pm 0.03 \mathrm{~mm}, 1.2 \pm 0.04 \mathrm{~mm}$ and $1.3 \pm 0.03 \mathrm{~mm}$ for red, yellow and green, respectively, which are in good agreement with the theoretical results calculated using the aplanatic principle and refraction theory (Figure 2e and Supplementary Fig. S6). Such a multifocus lens may be of use for certain optofluidic applications, such as the trapping of multiple cells at multispots. In contrast, a single focus lens (achromatic lens) is more attractive for many applications. It is well known that the achromatic lens can be constructed by the combination of a convex lens and FZP. The refractive convex lens can also be integrated by FSS-HFLM as described below. Then, multilayer integration of these optical components, which is possible using this technique, will enable fabrication of the achromatic lens. Excellent imaging characteristics are also obtained, as observed in Figure $2 \mathrm{f}-2 \mathrm{k}$. The object consists of the 5-mm letters 'RIKEN' placed $10 \mathrm{~mm}$ away from the backside of the microchip. The image is magnified by an objective lens and collected by aCD.

\section{High-quality integration of $3 \mathrm{D}$ refractive microlenses into glass microchannels}

Refractive microlenses and arrays are important for microfocusing, imaging, laser shaping and miniaturized cameras. These microlenses are more attractive for optofluidic applications ${ }^{34}$ because of their higher efficiency and resolution than FZPs. However, their 3D geometry leads to fabrication difficulties for integration inside an embedded microchannel. The FSS-HFLM technique overcomes this difficulty to permit integration of high-performance 3D refractive microlenses in a 3D glass microchannel. For example, Figure 3a-3b present a cross-sectional schematic and optical microscopy image of a refractive microlens with a $20-\mu \mathrm{m}$ radius and $10-\mu \mathrm{m}$ height integrated in 3D glass microfluidics. Due to the small size of the microlens, a 1$\mu \mathrm{m}$-thick flat scaffold is sufficient to eliminate the undulated channel 

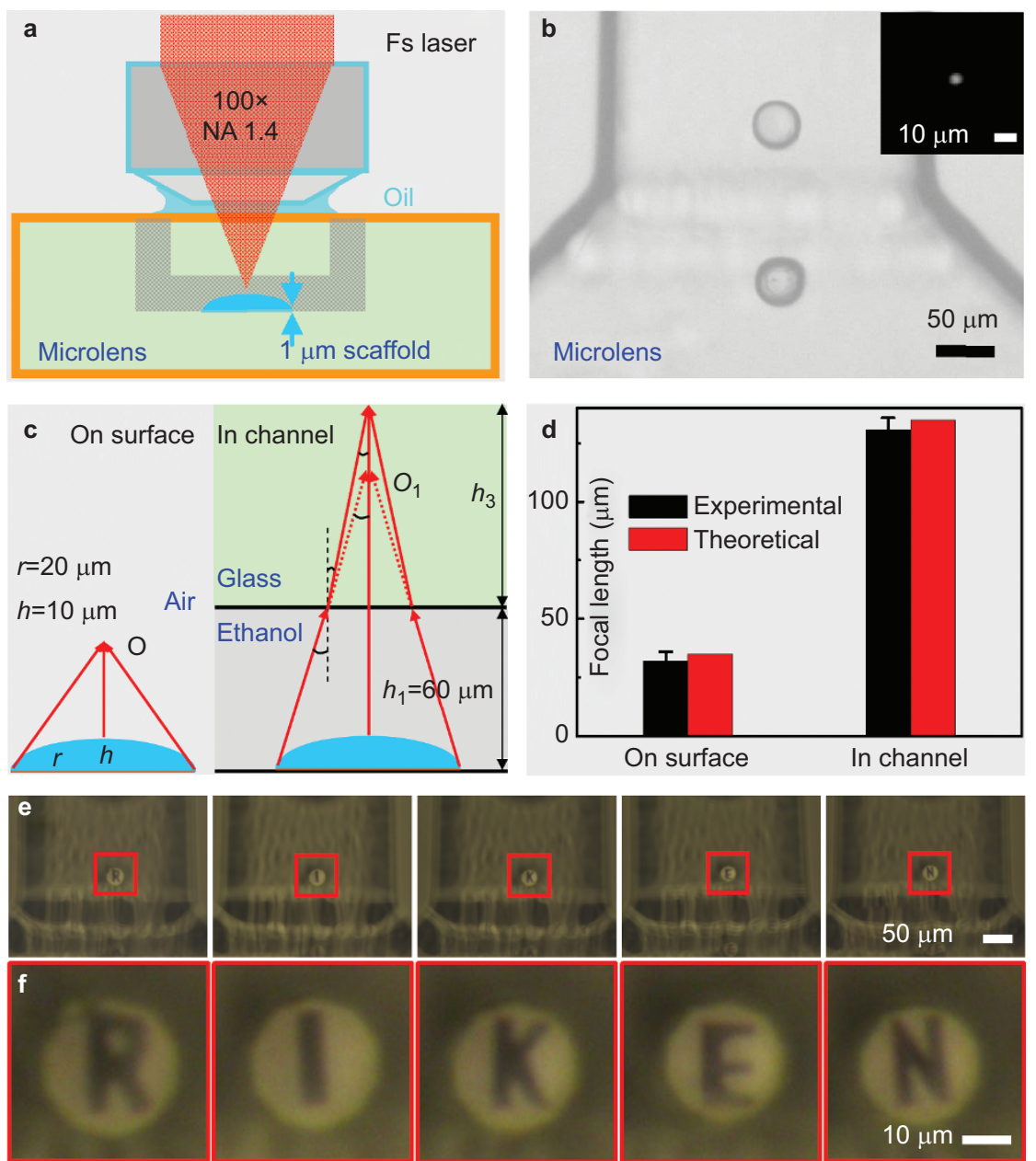

Figure 3 FSS-HFLM integration of a 3D refractive microlens into a 3D embedded glass microchannel. (a) Schematic of the FSS-HFLM integration. (b) Optical microscopy image of two polymer 3D microlenses integrated by FSS-HFLM into a channel. (c) Comparison of the focusing characteristics by 3D microlenses on the surface and in the channel, with different refractive index environments. (d) The $135 \pm 5 \mu \mathrm{m}$ focal length of the microlens in the channel is much longer than the $35 \pm 4 \mu \mathrm{m}$ focal length in air because the difference in the refractive indices between the polymer $\left(n_{\mathrm{su}}=1.58\right)$ and the solvent $\left(n_{\mathrm{ethanol}}=1.362\right)$ is much smaller than that between the polymer and air $\left(n_{\mathrm{air}}=1\right.$ ). These results agree with the theoretical values of $32 \mu \mathrm{m}$ in air and $131.6 \mu \mathrm{m}$ in ethanol (Supplementary Fig. S5). (e) Optical microscopy and (f) Magnified images of the letters 'RIKEN' using an integrated 3D microlens in a 3D embedded glass microchannel. 3D, three-dimensional; FSSHFLM, flat scaffold-supported hybrid femtosecond laser microfabrication; NA, Numerical aperture.

surface. The focal length of the microlens in the channel filled with ethanol is longer than that in air because the difference in the refractive index between the polymer $\left(n_{\mathrm{su} 8}=1.58\right)$ and solvent $\left(n_{\mathrm{ethanol}}=1.362\right)$ is smaller than that between the polymer and air $\left(n_{\text {air }}=1\right)$, as shown in Figure $3 \mathrm{c}$. The measured focal length in air is only $35 \pm 4 \mu \mathrm{m}$, while that in the microchannel filled with ethanol increases to as long as $135 \pm 5 \mu \mathrm{m}$ (Figure $3 \mathrm{~d}$ ). These results agree with the theoretical values of $32 \mu \mathrm{m}$ in air and $132 \mu \mathrm{m}$ in ethanol (Supplementary Fig. S7b). Under optical illumination, the 3D integrated microlens produced sharp focusing with a focal spot size of $2.9 \mu \mathrm{m}$ in diameter (the inset of Fig. $3 b$ and Supplementary Fig. S7a). When different letters are placed at the backside of the microchip at a distance of $10 \mathrm{~mm}$, clear imaging of every letter in 'RIKEN' is achieved, as demonstrated in Figure $3 \mathrm{e}$ and $3 \mathrm{f}$.

A 3D refractive microlens was directly fabricated in glass by FLAE. ${ }^{28}$ FLAE is categorized as a top-down fabrication process, in other words, subtractive manufacturing, because the laser irradiated regions are selectively removed by subsequent HF etching. However, its radius was $750 \mu \mathrm{m}$, much larger than the $20-\mu \mathrm{m}$ radius of the present polymer microlens, resulting in a longer focal length of $1.7 \mathrm{~mm}$.
Miniaturizing the microlens is not easy due to the low fabrication resolution $(\sim 10 \mu \mathrm{m})$, which is determined by the HF etching and second annealing $\left(\sim 630{ }^{\circ} \mathrm{C}\right)$ process. Such a low fabrication resolution also deteriorates the focusing performance $(\sim 30 \mu \mathrm{m}$ in diameter), while the polymer microlens produced a focal spot size of $2.9 \mu \mathrm{m}$ in diameter, as described above. TPP is categorized as a bottom-up fabrication process, in other words, additive manufacturing, and provides a high fabrication resolution (less than $100 \mathrm{~nm}$ ) by employing nonlinear absorption and the threshold effect.

FSS-HFLM integration of microlens arrays for coupling-free whitelight cell detection and counting

Site-selective cell detection and counting is highly desirable for biological research; however, there are few reports except for some demonstrations using optofluidics integrated with optical waveguides by femtosecond laser-induced refractive index modifications. ${ }^{6,15,35}$ Here, a system based on a new concept is presented by utilizing glass optofluidic chips integrated with polymer microlenses, as illustrated in Figure 4. High intensity is obtained at the focal spot of a microlens under white-light illumination when cells are not blocking the beam 
a Without cell pass
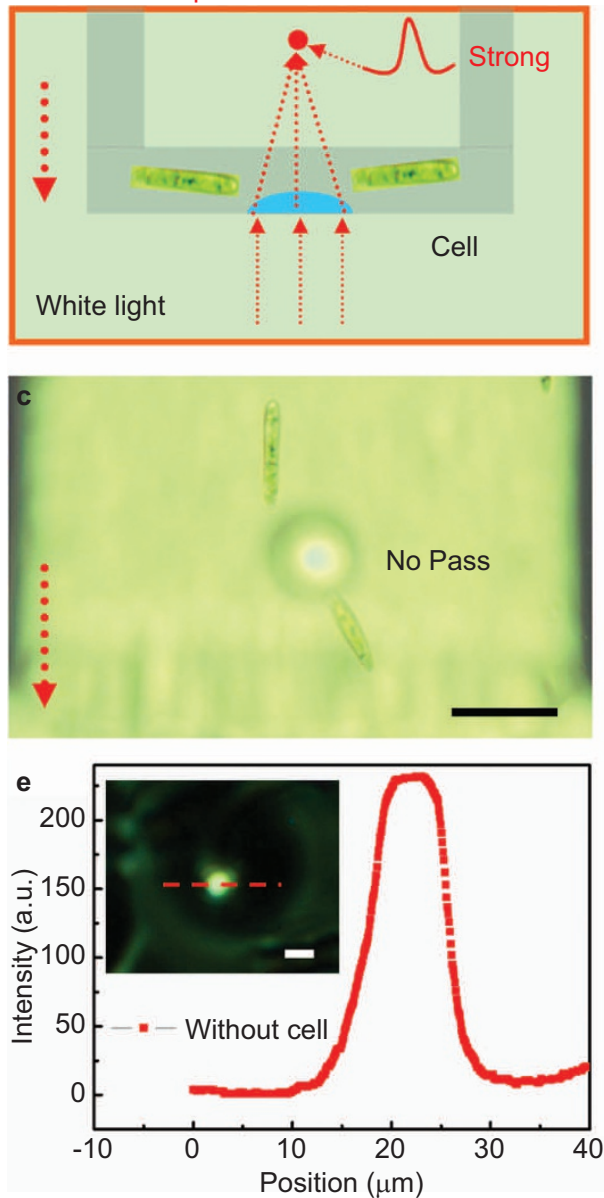

b with cell pass
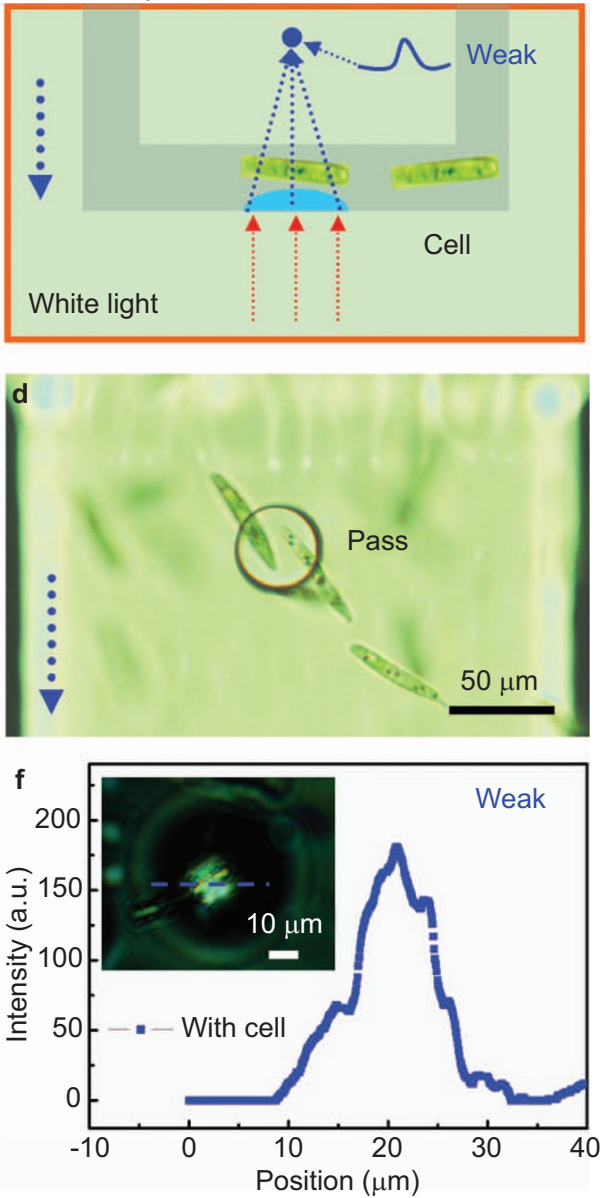

Figure 4 Optofluidic application for coupling-free white-light cell detection based on the intensity variations at the focal spot. Schematic principle of cell detection by an integrated optofluidic microchip. The intensity of the focal spot (a) in the absence of a cell is much stronger than (b) in the presence of a cell. Optical microscopy images of the 3D polymer microlens in a glass microchannel (c) without and (d) with the presence of a cell over the microlens. The 3D integrated optofluidic microchip is biocompatible with the cells, as demonstrated in Supplementary Movie 1. Comparison of the intensity variation at the focal spot of the microlens (e) without and (f) with a cell. The intensity at the focal spot decreases when the cell passes over the microlens due to reflection, scattering and absorption. 3D, three-dimensional.

(Figure 4a, 4c and 4e); however, a much lower intensity is observed as the cells move across the lens because the cells scatter, reflect and absorb the light (Figure $4 \mathrm{~b}, 4 \mathrm{~d}$ and $4 \mathrm{f}$ ). Thus, in situ cell detection is possible by monitoring the intensity variations. In addition, cell counting can be achieved by recording these variations as a function of the time, as demonstrated in Supplementary Fig. S8a. Each drop in intensity indicates a cell passing above the microlens. The magnitude of the intensity decrease varies between approximately $20 \%$ and $80 \%$, as the cells pass through different positions above the microlens (Supplementary Fig. S8b). Cells passing over the center of the lens result in the largest decline in intensity, whereas the decrease is smaller as the cells move farther away from the center. Some cells may miss the lens entirely because its $40-\mu \mathrm{m}$ diameter is much smaller than the channel width of $280 \mu \mathrm{m}$.

To monitor the entire channel along the width direction, an array of 7 lenses was integrated into the 3D glass microfluidics (upper-left in Figure 5a), and the variations of the intensity at the corresponding seven focal spots were examined (Figure 5a). The novelty of this device for optical biosensing is that the MLA enables in parallel detecting and counting cells with coupling-free white light. For the biosamples to be detected, 60 cells of Euglena, which is a popular flagellated laboratory microorganism living in freshwater environments and representing one of the simplest and earliest derived eukaryotic cells, ${ }^{36}$ were introduced. The optofluidics recorded 56 intensity drops, corresponding to a $93 \%$ success rate for detection. A small percentage of the cells $(\sim 7 \%)$ that were not detected most likely passed through the border areas between adjacent lenses.

Compared with state-of-the-art commercial flow focusing devices, ${ }^{37,38}$ the integrated optofluidic devices fabricated by the FSSHFLM method possess multifunctions, e.g., microfocusing/imaging and filtering/counting. Multifunction integration in a smaller-size microchip can greatly enhance the LOC functionalities for practical application. Therefore, a microfluidic system consisting of two arrays of seven refractive lenses and a $\mathrm{W}$-shape filter was designed and prepared for successive cell filtering and detection (Figure $5 \mathrm{~b}-5 \mathrm{e}$ and Supplementary Movie 2). The ' $W$ ' shape significantly increases the stability (Supplementary Fig. S9) to avoid deformation and breakdown during the development and characterization process. The dimension of the hole in the filter can be flexibly adjusted according to the sizes of the cells to be selectively separated from each other. For example, 7- $\mu \mathrm{m}$ holes are created, and cells of different sizes are introduced from the left in Figure 5d. Small cells with 50- $\mu \mathrm{m}$ lengths and 

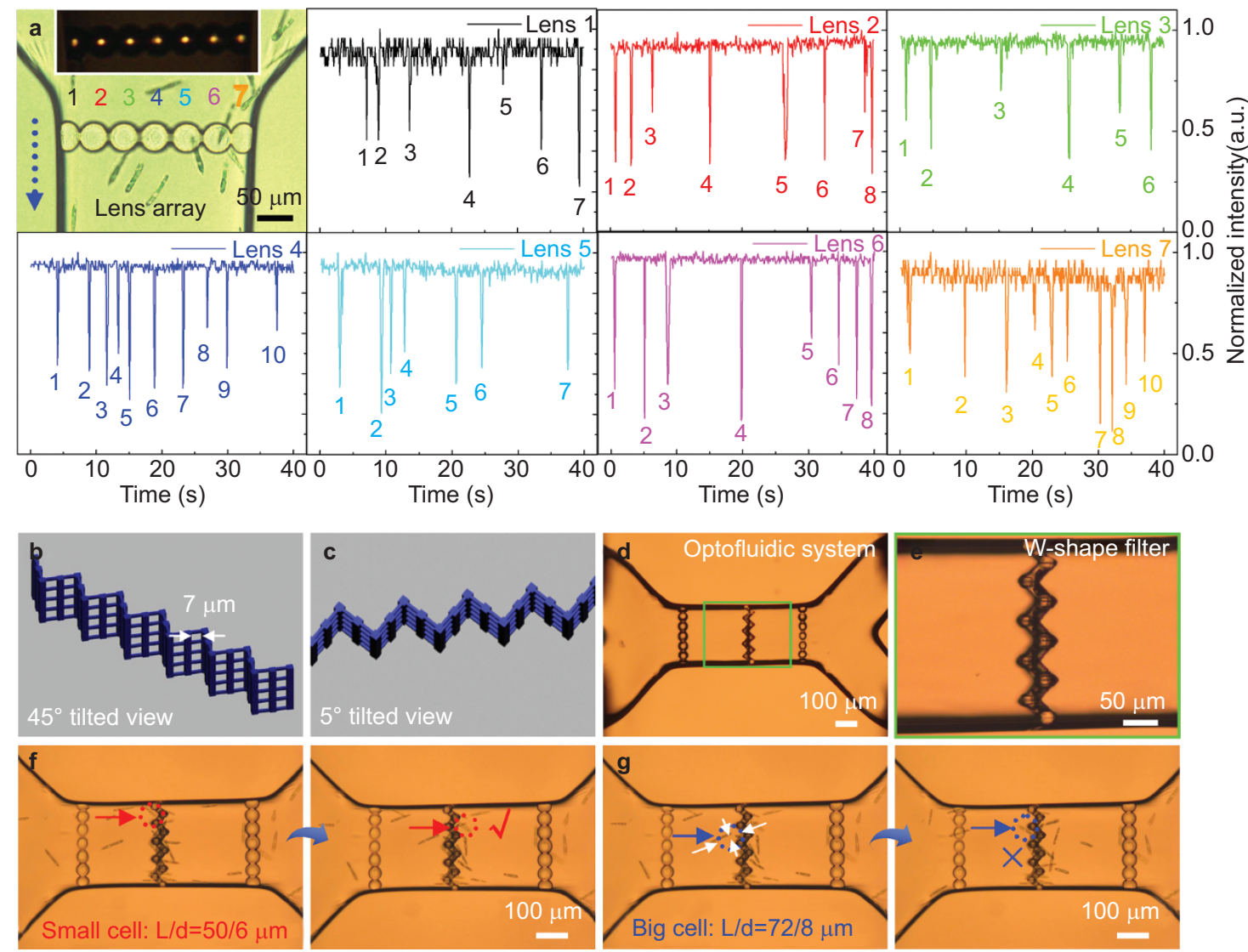

Figure 5 High-efficiency cell counting by designing and preparing optofluidic chips with an integrated 3D MLA. (a) An array of seven lenses was integrated into the microchannel to monitor cells passing through any location across the width of the channel. The inset presents an optical microscopy image of the focal spots produced by the seven lenses. The array achieves a success rate as high as $93 \%$ in cell detection by observing the time-dependent intensity changes from the seven microlenses. (b) $45^{\circ}$ and (c) $5^{\circ}$ tilted views of the W-shaped filters having 7 - $\mu \mathrm{m}$ holes. The ' $\mathrm{W}$ ' shape enhances the stability of the filter during the development process and during subsequent use. (d) Optical microscopy image of an optofluidic system consisting of two Y-shaped glass microchannels, two MLAs and a W-shaped filter. (e) Magnified image of a W-shaped filter. (f) A small cell with a 50- $\mu \mathrm{m}$ length and a 6 - $\mu \mathrm{m}$ width can pass through the W-filter and be detected by the right-hand microlens array. (g) Large cells with 72- $\mu \mathrm{m}$ lengths and 8 - $\mu \mathrm{m}$ widths are hindered by the W-filter (Supplementary Movie 2). 3D, threedimensional; MLA, microlens array.

6 - $\mu \mathrm{m}$ widths (specifically, $75 \% \pm 10 \%$ of them have widths less than $7 \mu \mathrm{m}$ ) freely pass through the filter and are detected by the microlens array along the right-hand side, while larger cells with $72-\mu \mathrm{m}$ lengths and $8-\mu \mathrm{m}$ widths are hindered by the $\mathrm{W}$-filter (as observed in Supplementary Movie 2). Compared with other optofluidic devices based on femtosecond laser-written optical waveguides, the present method has three advantages. First, this method does not require a complex and precise fiber-coupling system. Second, the microlens can focus white light and does not need a laser (Supplementary Fig. S4). Third, the microlens can be constructed in a microchannel made of various materials (e.g., PDMS, polymer or glass) because the resin is filled into the channel later for laser microfabrication and is subsequently washed out by the developer. In contrast, optical waveguides rely on the refractive index change of the glass substrate of the microfluidics induced by the femtosecond laser, such that the waveguides cannot be achieved in a material that is different from the substrate.

\section{CONCLUSIONS}

The present work has demonstrated in-channel integration of high-quality 2D-3D microoptical devices into 3D embedded glass microchannels in a spatially selective and designable manner by developing a new method-FSS-HFLM (Supplementary Table S1). The FSS-HFLM solves a key problem: the undulated surface that normally exists in PDMS, polymer, glass or silicon microfluidic channels is compensated by adding a layer of flat scaffold with an optimized thickness of 1-5 $\mu \mathrm{m}$ depending on the size of the microoptical components. As a proof of concept, two types of typical microoptical devices-2D diffractive FZPs and 3D refractive MLAs-were selectively fabricated in the channel. The FZP produced multicolor spots (red, yellow and green), and the focal length of an MLA in a microchannel filled with solvent was three times longer than that in air. Both of these devices showed good agreement with theoretical calculations. Moreover, the 2D FZP and 3D MLA exhibited excellent imaging performance of the letters 'RIKEN'. We further explored the application of $3 \mathrm{D}$ integrated optofluidic chips for coupling-free white-light cell counting. The success rate was as high as $93 \%$. Finally, microsystems integrated with two MLAs and a W-filter were designed and prepared for filtering and counting of cells with different sizes. In addition to the integration of 2D FZPs and 3D MLAs for biological detection, this FSS-HFLM method is highly promising for the integration of various $1 \mathrm{D}$ to $3 \mathrm{D}$ passive and active microoptical devices, including polymeric optical switches, wavelength-division multiplexing, on-chip microlasers and optical amplifiers for lab-on-a-chip 
optofluidic applications. We believe that this method will be applied to broader industrial applications by the further development of lowcost TPP using a continuous-wave laser $^{39}$ and new resin materials. ${ }^{40,41}$

\section{ACKNOWLEDGEMENTS}

This work was supported by JSPS KAKENHI Grant Number 25286038. The authors would like to thank Atsushi Miyawaki and Hiroyuki Kawano from the Laboratory for Cell Functions and Dynamics, Brain Science Institute, RIKEN for useful discussions and technical assistance.

1 Psaltis D, Quake SR, Yang C. Developing optofluidic technology through the fusion of microfluidics and optics. Nature 2006; 442: 381-386.

2 Fan X, White IM. Optofluidic microsystems for chemical and biological analysis. Nat Photonics 2011; 5: 591-597.

3 Schmidt H, Hawkins AR. The photonic integration of non-solid media using optofluidics. Nat Photonics 2011; 5: 598-604.

4 Li Z, Zhang Z, Emery T, Scherer A, Psaltis D. Single mode optofluidic distributed feedback dye laser. Opt Express 2006; 14: 696-701.

5 Wolfe DB, Conroy RS, Garstecki P, Mayers BT, Fischbach MA et al. Dynamic control of liquid-core liquid-cladding optical waveguides. Proc Natl Acad Sci USA 2004; 101: 12434-12438.

6 Bellini N, Vishnubhatla KC, Bragheri F, Ferrara L, Minzioni P et al. Femtosecond laser fabricated monolithic chip for optical trapping and stretching of single cells. Opt Express 2010; 18: 4679-4688.

7 Levy U, Campbell K, Groisman A, Mookherjea S, Fainman Y. On-chip microfluidic tuning of an optical microring resonator. Appl Phys Lett 2006; 88: 111107.

8 Vezenov DV, Mayers BT, Conroy RS, Whitesides GM, Snee PT et al. A low-threshold, high-efficiency microfluidic waveguide laser. J Am Chem Soc 2005; 127: 89528953.

9 Hoi SK, Chen X, Kumar VS, Homhuan S, Sow CH et al. A microfluidic chip with integrated colloidal crystal for online optical analysis. Adv Funct Mater 2011; 21: 2847-2853.

10 Lee SK, Park SG, Moon JH, Yang SM. Holographic fabrication of photonic nanostructures for optofluidic integration. Lab Chip 2008; 8: 388-391.

11 Kawata S, Sun HB, Tanaka T, Takada K. Finer features for functional microdevices. Nature 2001; 412: 697-698.

12 Maruo S, Nakamura O, Kawata S. Three-dimensional microfabrication with twophoton-absorbed photopolymerization. Opt Lett 1997; 22: 132-134.

13 Li L, Gattass RR, Gershgoren E, Hwang H, Fourkas JT. Achieving $\lambda / 20$ resolution by one-color initiation and deactivation of polymerization. Science 2009; 324: 910-913.

14 Gan Z, Cao Y, Evans RA, Gu M. Three-dimensional deep sub-diffraction optical beam lithography with $9 \mathrm{~nm}$ feature size. Nat Commun 2013; 4: 2061.

15 Ledermann A, Cademartiri L, Hermatschweiler M, Toninelli C, Ozin GA et al. Threedimensional silicon inverse photonic quasicrystals for infrared wavelengths. Nat Mater 2006; 5: 942-945.

16 Wang J, He Y, Xia H, Niu LG, Zhang R et al. Embellishment of microfluidic devices via femtosecond laser micronanofabrication for chip functionalization. Lab Chip 2010; 10: 1993-1996

17 Olsen MH, Hjorto GM, Hansen M, Met O, Svane IM et al. In-chip fabrication of freeform 3D constructs for directed cell migration analysis. Lab Chip 2013; 13: 48004809.

18 Lim TW, Son Y, Jeong YJ, Yang DY, Kong HJ et al. Three-dimensionally crossing manifold micro-mixer for fast mixing in a short channel length. Lab Chip 2011; 11: 100-103.

19 He Y, Huang BL, Lu DX, Zhao J, Xu BB et al. "Overpass" at the junction of a crossed microchannel: an enabler for 3D microfluidic chips. Lab Chip 2012; 12: 3866-3869.

20 Amato L, Gu Y, Bellini N, Eaton SM, Cerullo G et al. Integrated three dimensional filter separates nanoscale from microscale elements in a microfluidic chip. Lab Chip 2012; 12: $1135-1142$.
21 Sugioka K, Hanada Y, Midorikawa K. Three-dimensional femtosecond laser micromachining of photosensitive glass for biomicrochips. Laser Photonics Rev 2010; 4: 386-400.

22 Cheng Y, Sugioka K, Midorikawa K. Freestanding optical fibers fabricated in a glass chip using femtosecond laser micromachining for lab-on-a-chip application. Opt Express 2005; 13: 7225-7232.

23 Cheng Y, Sugioka K, Midorikawa K, Masuda M, Toyoda K et al. Three-dimensional micro-optical components embedded in photosensitive glass by a femtosecond laser. Opt Lett 2003; 28: 1144-1146.

24 Schaap A, Rohrlack T, Bellouard Y. Optical classification of algae species with a glass lab-on-a-chip. Lab Chip 2012; 12: 1527-1532.

25 Osellame R, Maselli V, Vazquez RM, Ramponi R, Cerullo G. Integration of optical waveguides and microfluidic channels both fabricated by femtosecond laser irradiation. Appl Phys Lett 2007; 90: 231118.

$26 \mathrm{Kim} \mathrm{M,} \mathrm{Hwang} \mathrm{DJ,} \mathrm{Jeon} \mathrm{H,} \mathrm{Hiromatsu} \mathrm{K,} \mathrm{Grigoropoulos} \mathrm{CP.} \mathrm{Single} \mathrm{cell} \mathrm{detection} \mathrm{using}$ a glass-based optofluidic device fabricated by femtosecond laser pulses. Lab Chip 2009; 9: 311-318.

27 Matsuo S, Kiyama S, Shichijo Y, Tomita T, Hashimoto S et al. Laser microfabrication and rotation of ship-in-a-bottle optical rotators. Appl Phys Lett 2008; 93: 051107.

28 Wang Z, Sugioka K, Midorikawa K. Three-dimensional integration of microoptical components buried inside photosensitive glass by femtosecond laser direct writing. Appl Phys A 2007; 89: 951-955.

29 Osellame R, Hoekstra HJ, Cerullo G, Pollnau M. Femtosecond laser microstructuring an enabling tool for optofluidic lab-on-chips. Laser Photonics Rev2011; 5: 442-463.

$30 \mathrm{Wu}$ D, Wu SZ, Xu J, Niu LG, Midorikawa K et al. Hybrid femtosecond laser microfabrication to achieve true 3D glass/polymer composite biochips with multiscale features and high performance: the concept of ship-in-a-bottle. Laser Photonics Rev 2014; 8: 458-467.

31 Wu D, Chen QD, Niu LG, Wang JN, Wang J et al. Femtosecond laser rapid prototyping of nanoshells and suspending components towards microfluidic devices. Lab Chip 2009; 9: 2391-2394.

32 Chen QD, Wu D, Niu LG, Wang J, Lin XF et al. Phase lenses and mirrors created by laser micronanofabrication via two-photon photopolymerization. Appl Phys Lett 2007; 91 : 171105.

33 Miyamoto K. The phase Fresnel lens. J Opt Soc Am 1961; 51: 17-20.

34 Erickson D, Sinton D, Psaltis D. Optofluidics for energy applications. Nat Photonics 2011; 5: 583-590.

35 Hanada Y, Sugioka K, Ishikawa IS, Kawano H, Miyawaki A. 3D microfluidic chips with integrated functional microelements fabricated by a femtosecond laser for studying the gliding mechanism of cyanobacteria. Lab Chip 2011; 11: 2109-2115.

36 Iseki M, Matsunaga S, Murakami A, Ohno K, Shiga K. A blue-light-activated adenylyl cyclase mediates photoavoidance in Euglena gracilis. Nature 2002; 415: 1047-1051.

37 Trujillo RR, Mills CA, Samitier J, Gomila G. Low cost micro-Coulter counter with hydrodynamic focusing. Microfluid Nanofluid 2007; 3: 171-176.

38 McClain MA, Culbertson CT, Jacobson SC, Ramsey JM. Flow cytometry of Escherichia coli on microfluidic devices. Anal Chem 2001; 73: 5334-5338.

39 Thiel M, Fischer J, Freymann GV, Wegener M. Direct laser writing of three-dimensional submicron structures using a continuous-wave laser at $532 \mathrm{~nm}$. Appl Phys Lett 2010; 97: 221102.

40 Malinauskas M, Farsari M, Piskarskas A, Juodkazis S. Ultrafast laser nanostructuring of photopolymers: a decade of advances. Phys Rep 2013; 533: 1-31.

41 Malinauskas M, Zukauskas A, Bickauskaite G, Gadonas R, Juodkazis S. Mechanism of three-dimensional structuring of photo-polymers by tightly focused femtosecond laser pulses. Opt Express 2010; 18: 10209-10211.

This work is licensed under a Creative Commons Attribution-

NonCommercial-NoDerivs 3.0 Unported License. The images or other third party material in this article are included in the article's Creative Commons license, unless indicated otherwise in the credit line; if the material is not included under the Creative Commons license, users will need to obtain permission from the license holder to reproduce the material. To view a copy of this license, visit http://creativecommons.org/licenses/ by-nc-nd/3.0/ 


\section{Corrigendum: In-channel integration of designable microoptical devices using flat scaffold-supported femtosecond-laser microfabrication for coupling-free optofluidic cell counting}

Dong Wu, Jian Xu, Li-Gang Niu, Si-Zhu Wu, Katsumi Midorikawa and Koji Sugioka

Light: Science \& Applications (2015) 4, e275; doi:10.1038/Isa.2015.48; published online 13 February 2015

Correction to: Light: Science \& Applications (2015) 4, e228; doi:10.1038/lsa.2015.1; published online 2 January 2015

In this paper, in the "Materials and methods" section, the type of laser from IMRA America, Inc. was wrongly given as Er-fiber. The correct type is $\mathrm{Yb}$-doped fiber.

We apologize for any inconvenience this may have caused. 